\title{
Using Sound to Represent Positional Accuracy of Address Locations
}

\author{
Nick Bearman and Andrew Lovett \\ School of Environmental Science \\ University of East Anglia \\ Norwich, NR4 7TJ, UK \\ n.bearman@uea.ac.uk; a.lovett@uea.ac.uk
}

\begin{abstract}
Uncertainty data is often ignored by spatial data users, for a number of reasons including difficulty of comprehension and limited methods of display. An extension to ArcGIS was created to allow the positional accuracy of address locations to be represented sonically using piano notes. This was evaluated by 49 spatial data users during a computerbased evaluation and subsequent discussion sessions. Two factors that had a significant impact on successful interpretation were the proportion of the attribute under investigation in the data and the presentation method. Knowledge of the data source also appeared important. Future studies will broaden the applications to include climate scenario data and virtual reality visualisations of future landscapes, as well as different aspects of sound.
\end{abstract}

Keywords: positional accuracy, sound, sonification, tone, ArcGIS

\section{LITERATURE REVIEW}

All spatial data have some uncertainty concerning their positional location. However, even when such characteristics are important in terms of fitness for use, they are sometimes ignored by users of the data. This study evaluated the use of sound to represent positional accuracy in Ordnance Survey's OS MasterMap ${ }^{\circledR}$ Address Layer 2 (AL2) data.

The AL2 data set has a number of status flags within it, including an indicator of positional accuracy. The latter specifies the accuracy in the positioning of an address point and is important because lower accuracy may mean addresses are shown in provisional locations up to several kilometres away from their true location. Ignoring such information could have serious consequences (e.g. for routing applications in emergency services usage). Despite this, a number of interviews with Ordnance Survey Account Managers and Pre and Post Sales staff suggested that often status flags are not properly considered when the data is used by external organisations.

The representation of uncertainty in spatial data has been widely discussed in the research literature and visual methods such as colour, blurring or multiple maps are most common (Appleton et al. 2004; Ehlschlaeger et al. 1997). However these methods can obscure underlying data or limit the amount of information shown. The use of other senses has been explored to address these limitations. Haptic (touch) maps are being developed, but they require specialised hardware and training to use (Golledge et al.,
2005). Sound is also being researched and the hardware required (sound card and headphones/speakers) is readily available, but user training is required (Pauletto \& Hunt, 2009).

One possible way of showing uncertainty information more effectively is to use sound in combination with vision, and this has been addressed from both a theoretical and practical point of view (Krygier, 1994; Fisher, 1994). MacVeigh \& Jacobson (2007) developed a prototype which sonified three different land uses (sea, land and harbour). They found participants understood the map quickly and that the sound enhanced their experience of the map. They suggested that an extension to an industry standard GIS (e.g. ESRI's ArcGIS) could be created which would use sound to represent spatial data. This integration into a commercial application would enable greater use and easier evaluation. Few of these ideas have gone beyond the proof of concept stage or had significant user testing. This field of research is still in an early stage of development and comparison is often difficult due to different terminologies and research frameworks (Frauenberger \& Stockman, 2009).

\section{METHODS}

An ArcScript (custom extension to ArcGIS) was created to allow evaluation of both visual and sonic techniques to represent positional accuracy with Ordnance Survey OS MasterMap ${ }^{\circledR}$ Topography and AL2 data for Norwich. The AL2 Positional Accuracy data were linked with the Topography data using the associated TOIDs (topographic identifiers).

The AL2 Positional Accuracy status flag values are: Surveyed (most accurate), Approximate, Postcode Unit Mean, Estimate and Postcode Sector Mean (least accurate) (Ordnance Survey, 2009). In a pilot study, participants said that five sounds were too many to understand effectively, as they were unable to discriminate between the different piano notes and found it difficult to relate the notes to the status flag values. The five categories were reduced to three by combining Postcode Sector Mean with Estimate and Approximate with Postcode Unit Mean. The data presented to participants were altered to allow different proportions of Surveyed values (see below). One section of the evaluation required a second dataset to be presented; this was fabricated "Council Tax bands" (local property value) information. 


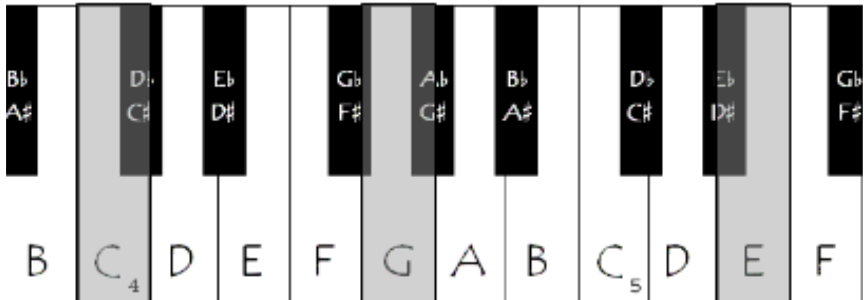

Figure 1. Piano keys with $\mathrm{C}_{4}, \mathrm{G}_{4}$ and $\mathrm{E}_{5}$ highlighted.

The three categories were represented using piano notes because the scale on a piano is very easy to visualise (i.e. participants can easily understand the difference between a 'high' and 'low' note) and the majority of people are familiar with this instrument. The notes chosen were $\mathrm{C}_{4}, \mathrm{G}_{4}$ and $\mathrm{E}_{5}$ which were based on the CEG triad split over two octaves (see Fig. 1). A triad was chosen because these are sets of notes which sound harmonious together (Burrus, 2009) and CEG was the favoured option in the pilot study. The highest note $\left(\mathrm{E}_{5}\right)$ represented the best level of accuracy and the lowest note $\left(\mathrm{C}_{4}\right)$ represented the poorest. These were played as the participant moved the mouse over the buildings, allowing them to either query a specific building or scan an area of data to get an overall view. A legend was provided, to allow the participant to link specific notes to specific values. The data set was shown to the participant using four different presentation methods (see Table I) and the topography layer was always shown as an outline. Sonic only (subsequently abbreviated to Sonic) showed this outline (see Fig. 2) and represented the AL2 Positional Accuracy using sound. Visual only (Visual) showed the topographical outline and the AL2 Positional Accuracy visually (see Fig. 3). Visual and Sonic Same (VS Same) used both sound and

TABLE I. THE FOUR DIFFERENT PRESENTATION METHODS (IN THE ORDER THEY WERE SHOWN TO THE PARTICIPANTS) AND WHICH DATA WERE SHOWN VISUALLY OR SONICALLY. THE TOPOGRAPHY LAYER WAS ALWAYS SHOWN VISUALLY.

\begin{tabular}{|l|l|l|l|}
\hline \multicolumn{1}{|c|}{ Presentation Method } & \multicolumn{1}{c|}{ Abbreviation } & \multicolumn{1}{c|}{ Visual Data } & \multicolumn{1}{c|}{ Sonic Data } \\
\hline Sonic only (see Fig. 2) & Sonic & Topography outlines only & AL2 Positional Accuracy \\
\hline Visual only (see Fig. 3) & Visual & AL2 Positional Accuracy & None \\
\hline Visual and Sonic representing the same variable & VS Same & AL2 Positional Accuracy & AL2 Positional Accuracy \\
\hline Visual and Sonic representing different variables & VS Different & Council Tax bands & AL2 Positional Accuracy \\
\hline
\end{tabular}

2. Project93.mxd - ArcMap - ArcInfo

File Edit View Bookmarks Insert Selection Iools Window $\underline{H}$ elp

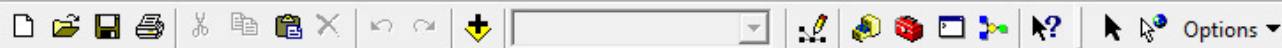

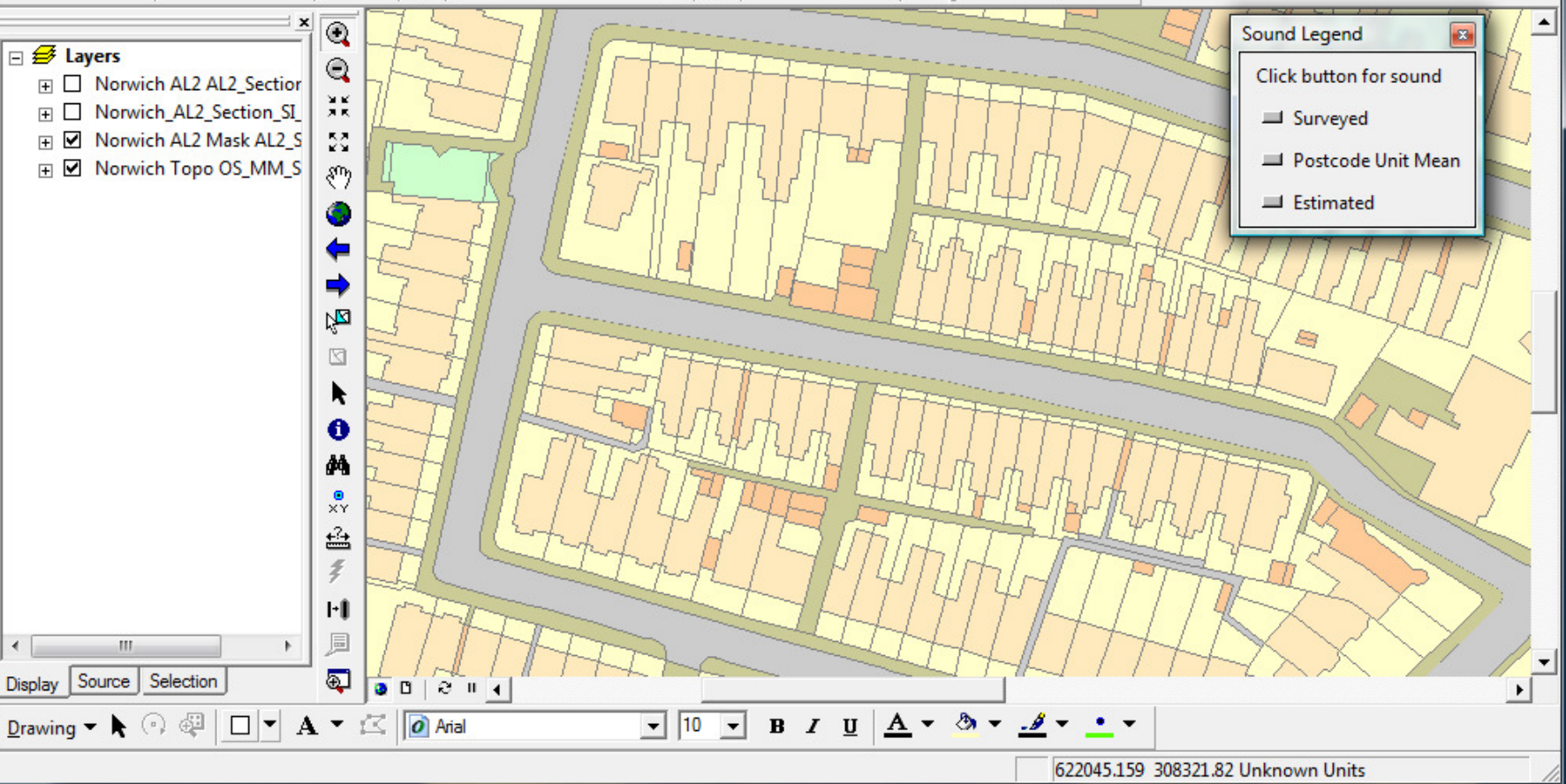

Figure 2. Screen shot of ArcGIS showing the data represented sonically. Note the topography layer shown visually, and the sound legend in the top right-hand corner. Ordnance Survey. (C) Crown Copyright. All rights reserved. 


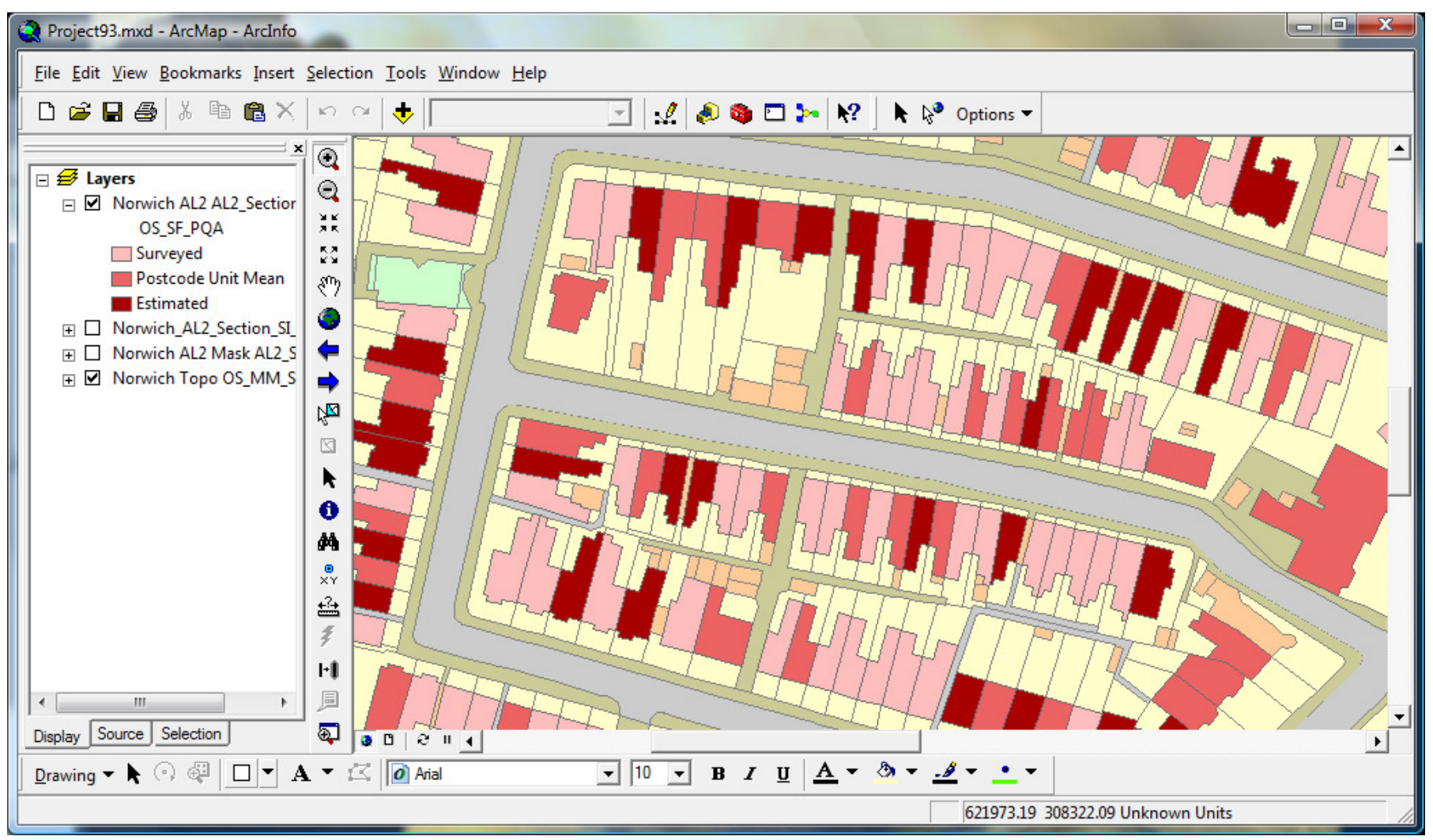

Figure 3. Screen shot of ArcGIS showing the data represented visually. Note the AL2 data shown visually, and the legend on the top left-hand side. The topography layer is also visible. Ordnance Survey. (C) Crown Copyright. All rights reserved.

visual methods to represent AL2 Positional Accuracy. Visual and Sonic Different (VS Different) used sound to represent AL2 Positional Accuracy and a visual method to represent Council Tax bands.

For each presentation method, the study participants were asked to identify the proportion of Surveyed values, from options of $25 \%, 50 \%$ or $75 \%$. The data were randomly assigned one of these values using a stratified random method for each presentation method. Other background questions were also asked, which allowed analysis depending on musical experience, learning preference and a number of other variables. This task was chosen because it combined a simple question (i.e. what proportion of the values are Surveyed) with the need to utilise sound in a way that visual representations are often employed.

A total of 49 participants completed the evaluation, consisting of 19 from Ordnance Survey, 23 from UEA and 7 from Local Authorities. All the participants had at least a basic knowledge of GIS, spatial data and ArcGIS and used these on a regular basis, although experience with AL2 varied. Headphones were used to provide auditory stimuli, with adjustable volume. The evaluation took place in groups of three to six, and was followed by a facilitated discussion for around 20 to 30 minutes which covered the participants' views and feelings and the potential uses of this technique.

\section{RESULTS \& DISCUSSION}

Nearly all participants (46 out of 49) identified the correct proportion of Surveyed values for 3 or 4 of the presentation methods. Fig. 4 shows how the mean answer for participants (correct $=1$, not correct $=0$ ) varied between proportion and presentation method, which were the two main influencing factors. VS Same and Visual methods (see Table I for abbreviations) performed best, while Sonic and VS Different had lower correct frequencies. The second broad trend was for more correct answers with $25 \%$ and $75 \%$

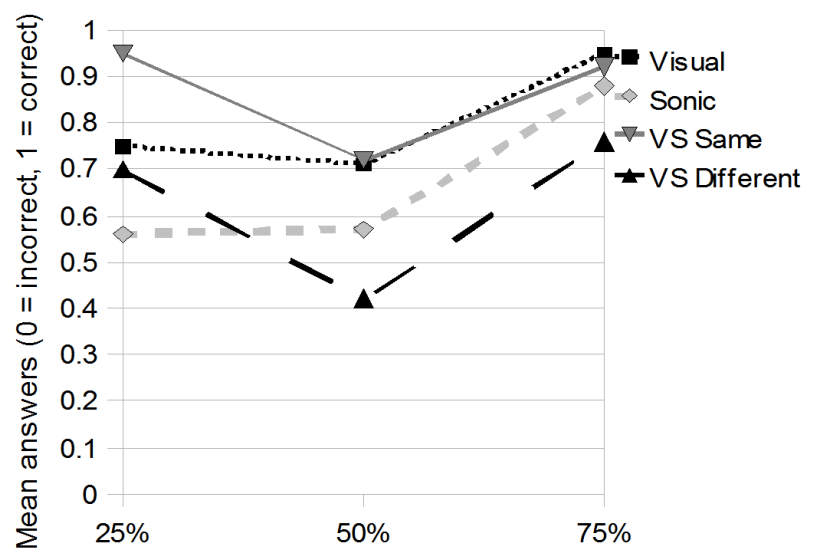

Proportion of Values with 'Surveyed'

Figure 4. Answers for all participants, split by presentation method and proportion. 
data proportions and fewer with a $50 \%$ proportion. The exception to this was the Sonic presentation method, which performed as badly with $25 \%$ as it did with $50 \%$. This may have occurred because the nature of the program made it fairly straightforward to find particular sounds when they were common, but more difficult when they were sparse, leading participants to overestimate the proportion. Both proportion $(\mathrm{p}<0.005)$ and presentation method $(\mathrm{p}<0.05)$ had a significant influence on whether a participant identified the correct proportion of Surveyed values.

A higher knowledge of the data set being sonified also increased the likelihood of the participants choosing the correct proportion. However, this trend was not so strong and although including it in a logistic regression model with proportion and presentation method improved the model (see Table II) the addition was not statistically significant.

The free text answers from the evaluation showed that some participants found the sonification very useful and that it added a large amount to the interpretation of the data, while others said the sound was very difficult to understand and when combined with vision, distracted them from the visual interpretation. Sonic and VS Different were considered harder to use than Visual and VS Same. Sonic only had a relatively low success rate and seems unlikely to be very successful with this type of interface.

The discussion sessions after each evaluation session provided further qualitative information and gave participants a chance to suggest changes and improvements to the technique. Preferences for the types of sounds used were subjective, and are likely to vary depending on the data set and the analysis taking place. A wider range of audio clips coupled with user choice could allow easier differentiation of sounds and potential for representation of a larger number of variables. Possibilities include different piano notes, different instruments, or completely different sounds, such as environmental or animal sounds. Colourblind users were highlighted as a group who might find the sonification useful; however a larger sample size is required to effectively evaluate this possibility.

The task chosen may limit the wider applicability of the results but there are few existing evaluations in this area so there is very little comparative data. The task needed to be easy enough to ensure that some of the participants managed to answer most/all of the questions correctly but not too difficult so that it would result in exclusively incorrect answers. Possible future options include more complex tasks (such as clustering exercises) and more comparisons of different presentation methods, utilising both sound and vision.

This research has highlighted specific characteristics that influence the ability of users to interpret sound to make proportion judgements. The proportion of the data the user is interested in and the presentation method are the two factors that appear to have the most impact on whether a person will be able to understand the information correctly. Knowledge of the data set being sonified also appears to have some impact, but this is not so clearly apparent with these results. These issues will be explored in future research which will
TABLE II. FACTORS ADDED TO THE LOGISTIC REGRESSION MODEL AND THEIR IMPACT

\begin{tabular}{|l|l|l|}
\hline Factors added to Model & $\mathbf{- 2}$ Log Likelihood & Cox \& Snell R $^{\mathbf{2}}$ \\
\hline Proportion & 182.01 & 0.043 \\
\hline Presentation Method & 168.579 & 0.11 \\
\hline Address Knowledge & 167.319 & 0.116 \\
\hline
\end{tabular}

evaluate the use of sound to represent uncertainty in UK climate scenario data and virtual reality visualisations of future landscapes.

\section{ACKNOWLEDGEMENTS}

The authors would like to thank all the participants in the study; the Ordnance Survey for their support, particularly Jenny Harding and Glen Hart for their advice, cooperation with data and assistance with arranging the evaluation sessions; and Katy Appleton for providing valuable comments while writing this extended abstract.

\section{REFERENCES}

Appleton, K., Lovett, A., Dockerty, T. \& Sünnenberg, G. (2004). Representing uncertainty in visualisations of future landscapes. In: Proceedings of the XXth ISPRS Congress.

Burrus, C. (2009). There's math behind the music! Available from: http://www.charlieburrus.com/MathInMusic/Index.htm [Accessed 17 February 2010].

Ehlschlaeger, C.R., Shortridge, A.M. \& Goodchild, M.F. (1997). Visualizing spatial data uncertainty using animation. Computers \& Geosciences. 23 (4), 387-395.

Fisher, P.F. (1994). Hearing the reliability in classified remotely sensed images. Cartography and Geographic Information Systems. 21 (1), 3136.

Frauenberger, C. \& Stockman, T. (2009). Auditory display design-an investigation of a design pattern approach. International Journal of Human-Computer Studies. 67 (11), 907-922.

Golledge, R.G., Rice, M. \& Jacobson, R.D. (2005). A commentary on the use of touch for accessing on-screen spatial pepresentations: the process of experiencing haptic maps and graphics. The Professional Geographer. 57 (3), 339-349.

Krygier, J.B. (1994). Sound and geographic visualization. In: A.M. MacEachern and D.R.F. Taylor (Eds) Visualization in Modern Cartography (pp. 149-166). Oxford: Elsevier Science.

MacVeigh, R. \& Jacobson, R.D. (2007). Increasing the dimensionality of a geographic information system (GIS) using auditory display. In: Proceedings of the 13th International Conference on Auditory Display.

Ordnance Survey (2009). OS MasterMap Address Layer 2 Technical Specification. Available from http://www.ordnancesurvey.co.uk/ oswebsite/products/osmastermap/layers/addresslayer2/detailedproduct infolal2 tech_spec.pdf [Accessed 9 March 2010].

Pauletto, S. \& Hunt, A. (2009). Interactive sonification of complex data. International Journal of Human-Computer Studies. 67 (11), 923-933. 